\title{
Ultrasonographic Characterization of Jaw Swellings- A Systematic Review
}

\author{
P.Sangeetha , G.Maragathavalli \\ Post Graduate Department Of Oral Medicine and Radiology Saveetha Dental College Saveetha University \\ HOD and Professor Of Oral Medicine and Radiology Saveetha Dental College Saveetha University, Chennai-77
}

Background:Various disease processes may affect head and neck regions, which present clinically as swellings. The disease processes which lead to such types of swellings can be broadly classified as inflammatory, cystic, benign or malignant in nature. In evaluation of jaw swellings, detailed case history and clinical examination are the most important and mandatory steps[1].

But in some cases, such as chronic inflammation, abscess formation, deep-seated or infected cystic lesion and neoplasm, clinical examination do not provide complete assessment of the exact origin and nature of swellings; such cases require radiological imaging. Therefore, to get a final diagnosis, clinical examination must be joined with various investigative procedures. Hence ultrasonographyused to diagnose the different kind of jaw swellings.[1]

In this review sensitivity, specificity, predictive value, and accuracy of the ultrasonography diagnosis were calculated in inflammatory, cystic, benign and malignant swellings.

Aim and Objective: To assess the reliability and accuracy of ulrasonography as a diagnostic aid in jaw swelling

To determine the accuracy, sensitivity,specificity and prediction values of ultrasound as means of diagnosis of jaw swellings.

Search strategy: The following electronic retrieval systems and databases were searched for identification of studies. The Cochrane Central Register of Controlled Trials (CENTRAL) PUBMED, MEDLINE, SCIENCE DIRECT.

Search criteria: Studies conducted in human study with clinical parameters which evaluated in the jaw swellings based on their echo intensity of jaw swellings.

Main results: Four studies were included in this review among all fourstudies, two studies only determined the accuracy, sensitivity,specificity and predictive values of ultrasonography diagnosis in jaw swellings. One study determined only the sensitivity and specificity; another study determined only the percentage of ultrasonography diagnosis.

Conclusion: Ultrasonography can be used as a diagnostic aid in jaw swellings.But Quality studies which assesing the diagnostic accuracy, sensitivity and specificity are less.To conclude, quality studies are needed to establish whether the ultrasonography diagnosis is accurate in all jaw swellings.

\section{Introduction}

Various disease processes may affect head and neck regions, which present clinically as swellings. The disease processes which lead to such types of swellings can be broadly classified as inflammatory, cystic, benign or malignant in nature. In evaluation of jaw swellings, detailed case history and clinical examination are the most important and mandatory steps.[1]

But in some cases, such as chronic inflammation, abscess formation, deep-seated or infected cystic lesion and neoplasm, clinical examination do not provide complete assessment of the exact origin and nature of swellings; such cases require radiological imaging. Therefore, to get a final diagnosis, clinical examination must be joined with various investigative procedures[1]

The physical examination of jaw swellings lacks the diagnostic accuracy hence various investigations been introduced to evaluate the jaw swellings the ultrasonography been one of the recent tools.[2]

Ultrasonography has several advantages over other modalities as it is harmless, uses no ionizing radiation,is widely available, easy-to-use, non-invasive, in expensive and unaffected by metal artefacts such as dentalrestorations. It can be performed without heavy sedation.Ultrasound causes no health problems and maybe repeated as often as necessary.[4]

The sonographic images are identified in the terms of echoes as hypoechoic,hyperechoic and anechoic a mass is hypoechoic it has a intensity lower than that of the adjacent tissues, hyperechoic is used for the mass of higher intensity, andisoechoic is used for the masses shows intensity similar to that of adjacent tissues. [4] 


\section{Structured Question}

Are ultrasounds accurate in diagnosing the different varieties of jaw swellings?

What is specificity and sensitivity of ultrasound in diagnosing the jaw swellings?

\section{Materials And Methods}

\section{Source}

A comprehensive literature search of the following databases were done which included studies of The Cochrane Central Register of Controlled Trials (CENTRAL)

PUBMED

MEDLINE

SCIENCE DIRECT

We also searched websites of products manufactures, as well as Google scholar.

PUBMED

Search Methodology: (PUBMED)

\begin{tabular}{|c|c|c|c|c|}
\hline Search & Add to builder & Query & Items found & Time \\
\hline$\# 39$ & Add & Search (((\#32) AND \#33) AND \#34) AND \#35 Filters: Humans; English & $\underline{85}$ & 06:15:32 \\
\hline$\# 38$ & Add & Search (((\#32) AND \#33) AND \#34) AND \#35 Filters: Humans & 100 & $06: 13: 22$ \\
\hline$\# 36$ & Add & Search (((\#32) AND \#33) AND \#34) AND \#35 & 105 & 06:08:49 \\
\hline$\# 35$ & Add & Search $(((((((\# 23)$ OR \#24) OR \#25) OR \#26) OR \#27) OR \#28) OR \#29) OR \#30 & 1261984 & 05:59:09 \\
\hline$\# 34$ & Add & Search $((((()((\# 14)$ OR \#15) OR \#16) OR \#17) OR \#18) OR \#19) OR \#20) OR \#21) OR \#22 & $\underline{4054807}$ & 05:58:14 \\
\hline$\# 33$ & Add & 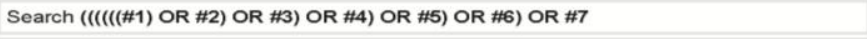 & $\underline{93349}$ & 05:56:37 \\
\hline$\# 32$ & Add & Search $((((((\#)$ OR \#9) NOT \#11) OR \#11) OR \#13) NOT \#13) OR \#10) OR \#12 & $\underline{399569}$ & 05:54:47 \\
\hline$\# 30$ & Add & Search sensitivity analysis & 403965 & 05:22:19 \\
\hline$\# 29$ & Add & Search sensitivity specificity & $\underline{432285}$ & 05:21:34 \\
\hline \#28 & Add & Search specificity & $\underline{860826}$ & 05:21:20 \\
\hline$\# 27$ & Add & Search specificity diagnosis & $\underline{444504}$ & 05:21:03 \\
\hline$\# 26$ & Add & Search specificity sensitivity & 432285 & $05: 20: 31$ \\
\hline$\# 25$ & Add & Search sensitivity & $\underline{826901}$ & 05:19:42 \\
\hline$\# 24$ & Add & Search echogenic & $\underline{3338}$ & 05:19:25 \\
\hline$\# 23$ & Add & Search echo intensity & 4604 & 05:19:05 \\
\hline$\# 22$ & Add & Search diagnostic radiology & $\underline{889755}$ & 05:18:21 \\
\hline$\# 21$ & Add & Search oral radiology & $\underline{5781}$ & 05:17:56 \\
\hline$\# 20$ & Add & Search dental radiology & $\underline{26369}$ & $05: 17: 23$ \\
\hline$\# 19$ & Add & Search histopathologic & $\underline{37286}$ & 05:17:02 \\
\hline$\# 18$ & Add & Search diagnostic histopathology & $\underline{6243}$ & 05:16:08 \\
\hline$\# 17$ & Add & Search histopathological diagnosis & $\underline{51654}$ & $05: 15: 49$ \\
\hline$\# 16$ & Add & Search histopathology & $\underline{2324662}$ & 05:15:34 \\
\hline$\# 15$ & Add & Search clinical diagnostic & 1508370 & 05:15:09 \\
\hline$\# 14$ & Add & Search clinical diagnos is & $\underline{1611801}$ & $05: 14: 27$ \\
\hline$\# 13$ & Add & Search ultrasonographic evaluation & $\underline{4273}$ & $05: 13: 28$ \\
\hline$\# 12$ & Add & Search ultrasonographic & 16544 & 05:13:12 \\
\hline$\# 11$ & Add & Search ultrasonography diagnosis & $\underline{322484}$ & $05: 12: 45$ \\
\hline$\# 10$ & Add & Search ultrasonography & $\underline{328057}$ & 05:12:35 \\
\hline \#9 & Add & Search ultrasound imaging & $\underline{339456}$ & $05: 12: 14$ \\
\hline$\# 8$ & Add & Search ultrasound & $\underline{396594}$ & 05:12:03 \\
\hline$\# 7$ & Add & Search mandible & $\underline{55833}$ & $05: 11: 30$ \\
\hline \#6 & Add & Search maxilla & $\underline{28913}$ & 05:11:14 \\
\hline$\# 5$ & Add & Search malignant swellings & 1890 & 05:10:36 \\
\hline$\# 4$ & Add & Search benign swellings & 1316 & 05:10:13 \\
\hline$\# 3$ & Add & Search cystic swellings & $\underline{2169}$ & 05:09:53 \\
\hline \#2 & Add & Search inflammatory swellings & 14274 & 05:09:33 \\
\hline$\# 1$ & Add & Search jaw swellings & $\underline{506}$ & $05: 08: 56$ \\
\hline
\end{tabular}

Search Methodology: (MESH) 


\begin{tabular}{|c|c|c|c|c|}
\hline \multicolumn{3}{|l|}{ History } & \multicolumn{2}{|c|}{ Clear history } \\
\hline Search & Add to builder & Query & Items found & Time \\
\hline$\# 67$ & Add & Search ((\#64) AND \#65) AND \#66 Filters: Humans; English & $\underline{81}$ & 23:58:52 \\
\hline$\# 66$ & Add & Search ((\#51) OR \#54) OR \#57 Filters: Humans; English & $\underline{50874}$ & 23:58:13 \\
\hline$\# 65$ & Add & Search (((\#31) OR \#36) OR \#40) OR \#42 Filters: Humans; English & 131227 & 23:57:30 \\
\hline$\# 64$ & Add & Search (((\#15) OR \#13) OR \#19) OR \#27 Filters: Humans; English & 168922 & 23:56:48 \\
\hline$\# 57$ & Add & Search "Jaw Diseases"[Mesh] & $\underline{78591}$ & 23:44:56 \\
\hline$\# 54$ & $\underline{\text { Add }}$ & Search "Jaw Cysts"[Mesh] & $\underline{6495}$ & 23:44:11 \\
\hline$\# 51$ & $\underline{\text { Add }}$ & Search "Jaw Neoplasms"[Mesh] & 17160 & 23:43:14 \\
\hline$\# 44$ & Add & Search "Edema"[Mesh] & $\underline{32957}$ & $23: 40: 43$ \\
\hline$\# 42$ & Add & Search "Mandible"[Mesh] & $\underline{41728}$ & 23:39:57 \\
\hline$\# 40$ & Add & Search "Maxilla"[Mesh] & $\underline{21179}$ & 23:39:32 \\
\hline$\# 36$ & Add & Search "Jaw"[Mesh] & $\underline{79468}$ & 23:38:48 \\
\hline \#31 & Add & Search "Head"[Mesh] & $\underline{147842}$ & 23:37:41 \\
\hline$\# 27$ & Add & Search "Ultrasonography, Doppler, Color"[Mesh] & $\underline{14698}$ & 23:36:42 \\
\hline$\# 19$ & $\underline{\text { Add }}$ & Search "Ultrasonography, Doppler, Duplex"[Mesh] & $\underline{19009}$ & 23:35:54 \\
\hline$\# 13$ & Add & Search "Ultrasonography, Doppler"[Mesh] & $\underline{51220}$ & 23:31:55 \\
\hline$\# 15$ & Add & Search "Ultrasonography"[Mesh] & $\underline{228562}$ & 23:27:37 \\
\hline
\end{tabular}

Search Methodology: (SCIENCE DIRECT)

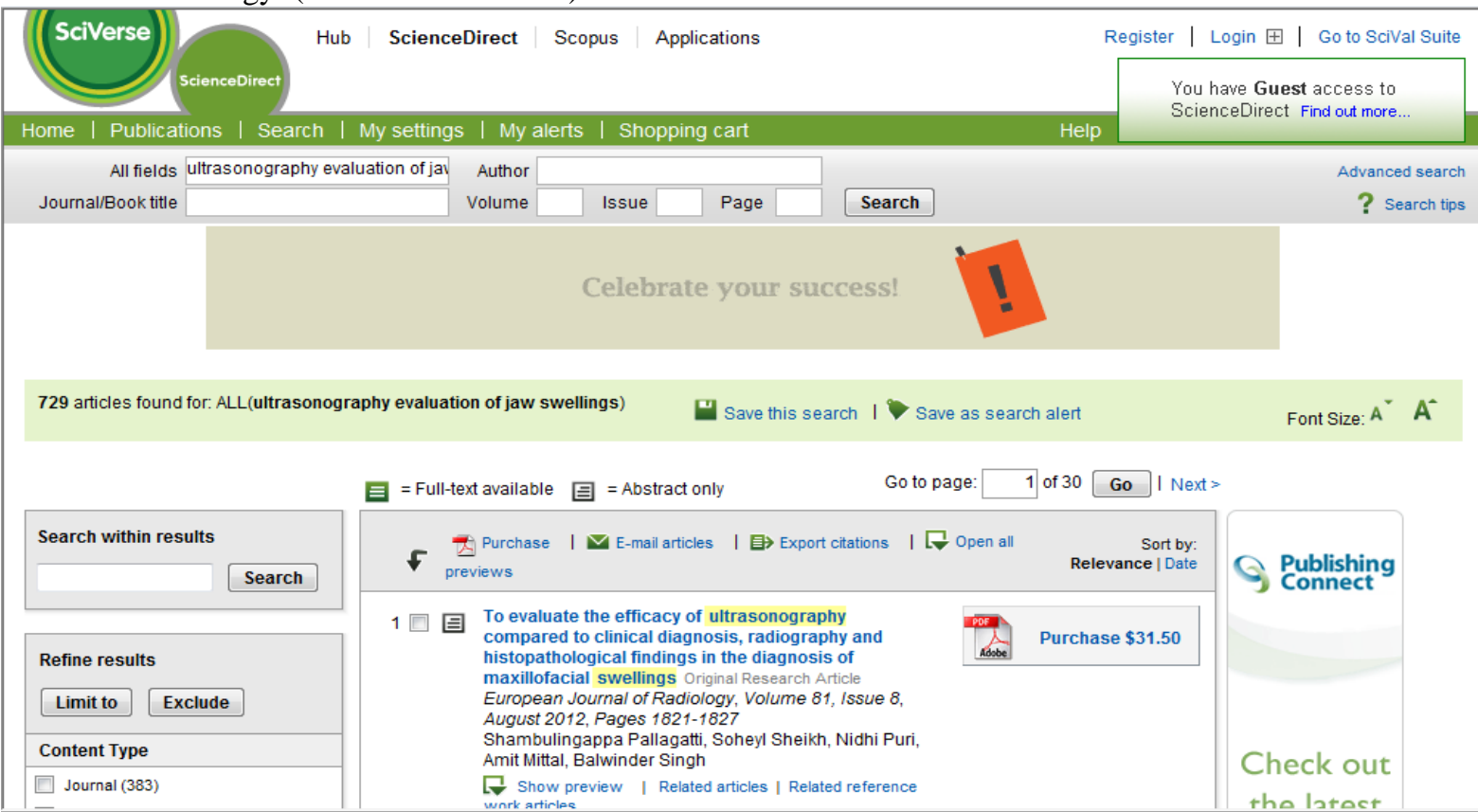

Criteria for considering studies for this review

We included studies in which diagnosis are made underultrasonography in jaw swellings. The main inclusion entering being Clinical

Criteria for included studies for this review

Clinical trial

Any age groups

Jaw swellings maxilla and mandible

The articles are excluded according to following criteria

Case reports and review articles

Neck swellings

Search flow chart 


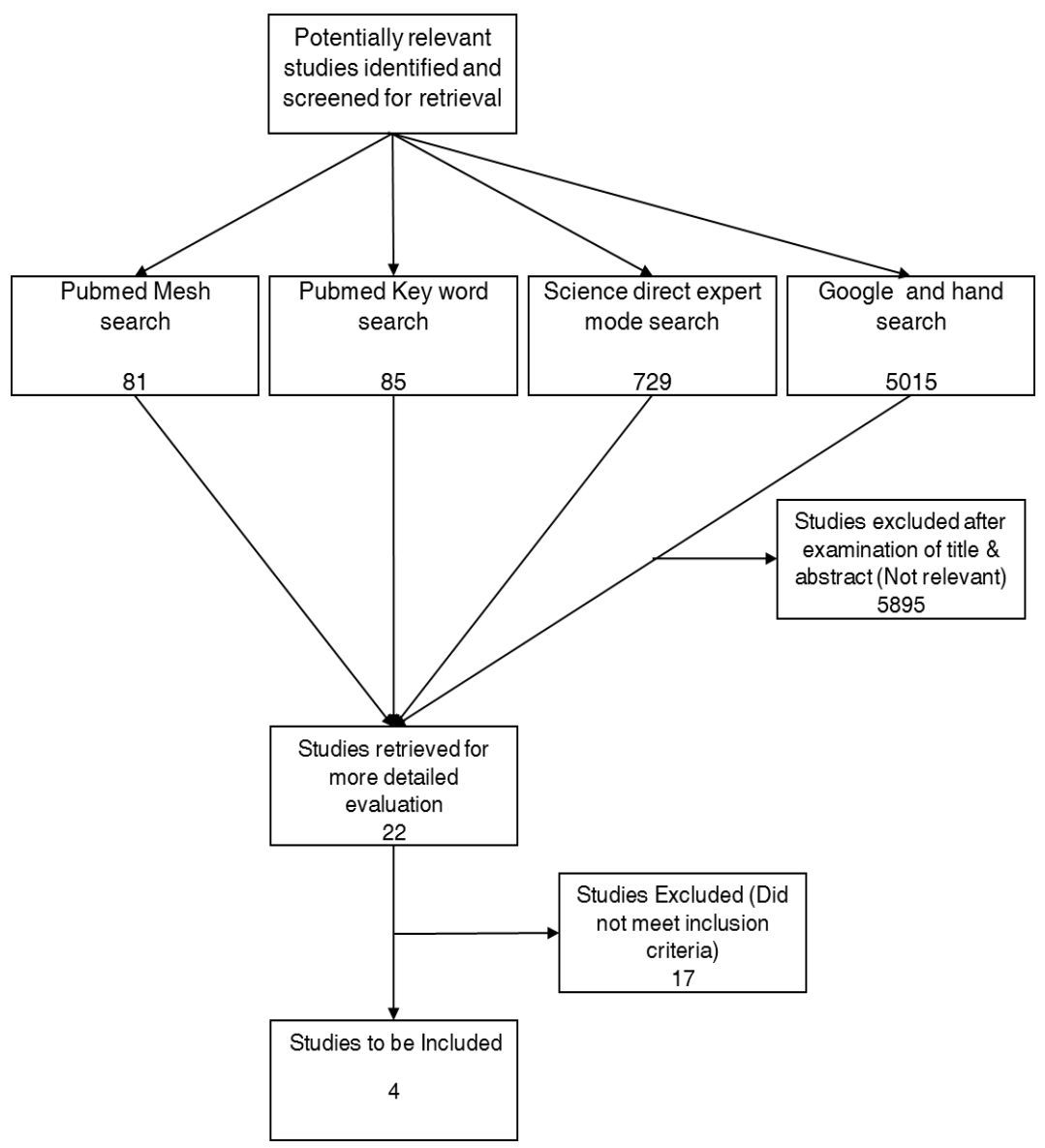

VARIABLES OF INTEREST TABLE

\begin{tabular}{|c|c|c|}
\hline \multirow{8}{*}{$\begin{array}{l}\text { ACCURACY } \\
\text { OF USG } \\
\text { EVALUATED }\end{array}$} & SHAPE & Oval, round, lobular, polygonal, irregular, \\
\hline & BOUNDARY & $\begin{array}{l}\text { Very clear, relatively clear, partialy unclear, ill } \\
\text { defined }\end{array}$ \\
\hline & ECHO INTENSITY & Anechoic, isoechoic, hypoechoic, hyperechoic, mixed \\
\hline & $\begin{array}{l}\text { USG } \\
\text { ARCHTECHTURE }\end{array}$ & Homogenous, heterogenous \\
\hline & NECROSIS & Ecentric, central \\
\hline & CAICIFTCATtoN & Macrocalcification, microcalcification \\
\hline & $\begin{array}{l}\text { POATERTOR } \\
\text { ECHOES }\end{array}$ & Enhanced, unchanged, attenuated, \\
\hline & $\begin{array}{l}\text { CHARACTERISTIC } \\
\mathrm{s}\end{array}$ & Cystic, solid, mixed \\
\hline SENSITIVITY & & \\
\hline SPECIFICITY & & \\
\hline $\begin{array}{l}\text { POSITIVE } \\
\text { PREDICTION }\end{array}$ & & \\
\hline $\begin{array}{l}\text { NEGATIVE } \\
\text { PREDICTION }\end{array}$ & & \\
\hline
\end{tabular}

Data Extraction Form

A Standardized data extraction form was used to retrieve the data from the selected articles. Citation Of Author 
Name Of The Author

Year Of Publication

Sample size

Type of lesion -Inflammatory, cystic, benign and malignant swellings

Echo intensity hyperechoic, hypoechoic and anechoic

Shape

Boundary

USG Architecture

Necrosis

Calcification

Posterior echoes

Characteristics

\section{Results}

General Information of Study Characteristics

\begin{tabular}{|c|c|c|c|c|c|c|c|c|c|c|c|c|c|}
\hline S.NO & AUTHOR & YEAR & TYPE OF LESION & $\begin{array}{l}\text { SAMPLE } \\
\text { SIZE }\end{array}$ & $\begin{array}{l}\mathrm{ACC} \\
\mathrm{EV}\end{array}$ & $\begin{array}{l}\text { CURA } \\
\text { ALUA }\end{array}$ & ACY & $\begin{array}{l}\mathrm{OF} \\
\mathrm{D}(\mathrm{S})\end{array}$ & $\begin{array}{l}\text { USG } \\
M / N\end{array}$ & & & & $\begin{array}{l}\text { USG } \\
\text { COMPARED }\end{array}$ \\
\hline I. & R.Chandak & 2011 & $\begin{array}{l}\text { INFLAMMATORY } \\
\text { CYSTIC }\end{array}$ & 70 & M & M & $\mathrm{M}$ & M & M & $\mathbf{M}$ & $\mathrm{M}$ & $\mathrm{M}$ & $\mathrm{CD}$ \\
\hline & & & $\begin{array}{l}\text { BENIGN } \\
\text { MALIGNANT }\end{array}$ & & $\mathrm{S}$ & B & $\mathrm{E}$ & $\mathrm{A}$ & $\mathrm{N}$ & $\mathrm{C}$ & $\mathrm{P}$ & $\mathrm{C}$ & \\
\hline II & shivanand & 2010 & $\begin{array}{l}\text { INFLAMMATORY } \\
\text { CYSTIC } \\
\text { BENIGN } \\
\text { MALIGNANT }\end{array}$ & 40 & $\begin{array}{l}\mathrm{N} \\
\mathrm{M}\end{array}$ & $\begin{array}{l}\mathrm{N} \\
\mathrm{M}\end{array}$ & $\mathrm{M}$ & $\begin{array}{l}\mathrm{N} \\
\mathrm{M}\end{array}$ & $\begin{array}{l}\mathrm{N} \\
\mathrm{M}\end{array}$ & $\begin{array}{l}\mathbf{N} \\
\mathrm{M}\end{array}$ & $\begin{array}{l}\mathbf{N} \\
\mathbf{M}\end{array}$ & $\begin{array}{l}\mathrm{N} \\
\mathrm{M}\end{array}$ & $\mathrm{CD} / \mathrm{HIS}$ \\
\hline III & K. Srinivas & 2009 & INFLAMMATORY & 25 & M & M & M & $\begin{array}{l}\mathrm{N} \\
\mathrm{M}\end{array}$ & $\begin{array}{l}\mathrm{N} \\
\mathrm{M}\end{array}$ & $\begin{array}{l}\mathrm{N} \\
\mathrm{M}\end{array}$ & $\begin{array}{l}\mathrm{N} \\
\mathrm{M}\end{array}$ & $\begin{array}{l}\mathrm{N} \\
\mathrm{M}\end{array}$ & $\mathrm{CD}$ \\
\hline IV & B.OAkinbami & 2006 & $\begin{array}{l}\text { CYSTIC } \\
\text { BENIGN } \\
\text { MALIGNANT }\end{array}$ & 76 & M & M & $\mathrm{M}$ & M & $\mathbf{M}$ & $\mathbf{M}$ & $\mathbf{M}$ & M & HIS \\
\hline $\mathrm{S}=\mathrm{Sha}$ & & & $\mathrm{M}=\mathrm{Ment}$ & & & & & & & & & & \\
\hline $\mathrm{B}=\mathrm{Bou}$ & dary & & $\mathrm{NM}=\mathrm{Not}$ & entioned & & & & & & & & & \\
\hline $\mathrm{E}=\mathrm{Ech}$ & Intensity & & & & & & & & & & & & \\
\hline$A=A r c$ & itecture & & $\mathrm{CD}=\mathrm{Clin}$ & Dignosis & & & & & & & & & \\
\hline $\mathrm{N}=\mathrm{Nec}$ & osis & & $\mathrm{HIS}=\mathrm{Hist}$ & athology & & & & & & & & & \\
\hline $\mathrm{C}=\mathrm{Cal}$ & fication & & & & & & & & & & & & \\
\hline $\mathrm{P}=\mathrm{Pos}$ & Ior Echoes & & & & & & & & & & & & \\
\hline Cha & torictio & & & & & & & & & & & & \\
\hline
\end{tabular}


GENERAL INFORMATION OF STUDY RESULTS

\begin{tabular}{|c|c|c|c|c|c|c|c|c|c|c|c|c|c|c|c|c|c|}
\hline \multirow[t]{2}{*}{ AUTHOR } & & \multicolumn{4}{|c|}{ ECHO INTENSITY \% } & \multicolumn{4}{|c|}{ ACCURACY \% } & \multicolumn{4}{|c|}{ SENSITIVITY \% } & \multicolumn{4}{|c|}{ SPECIFICITY \% } \\
\hline & & I & $\mathrm{C}$ & B & M & I & $\mathrm{C}$ & B & M & I & $\mathrm{C}$ & B & M & I & $\mathrm{C}$ & B & M \\
\hline \multirow[t]{5}{*}{$\begin{array}{l}\text { R.CHANDA } \\
\mathrm{K}\end{array}$} & A & $\begin{array}{l}31 . \\
4\end{array}$ & $\begin{array}{l}87 . \\
5\end{array}$ & $\begin{array}{l}22 . \\
2\end{array}$ & & & & & & & & & & & & & \\
\hline & I & $\begin{array}{l}17 . \\
1\end{array}$ & & & & $\begin{array}{l}98 . \\
5\end{array}$ & $\begin{array}{l}98 . \\
5\end{array}$ & $\begin{array}{l}98 . \\
5\end{array}$ & $\begin{array}{l}98 . \\
5\end{array}$ & $\begin{array}{l}97 . \\
1\end{array}$ & $\begin{array}{l}10 \\
0\end{array}$ & $\begin{array}{l}10 \\
0\end{array}$ & 100 & $\begin{array}{l}10 \\
0\end{array}$ & $\begin{array}{l}98 . \\
3\end{array}$ & $\begin{array}{l}98 . \\
3\end{array}$ & $\begin{array}{l}98 . \\
0\end{array}$ \\
\hline & $\begin{array}{l}\mathrm{H} \\
\mathrm{O}\end{array}$ & $\begin{array}{l}42 . \\
8\end{array}$ & $\begin{array}{l}12 . \\
5\end{array}$ & $\begin{array}{l}33 . \\
3\end{array}$ & $\begin{array}{l}22 . \\
2\end{array}$ & & & & & & & & & & & & \\
\hline & $\mathrm{H}$ & & & $\begin{array}{l}33 . \\
3\end{array}$ & 77 & & & & & & & & & & & & \\
\hline & M & 8.5 & & $\begin{array}{l}11 . \\
1\end{array}$ & $\begin{array}{l}55 . \\
7\end{array}$ & & & & & & & & & & & & \\
\hline \multicolumn{18}{|l|}{$\begin{array}{l}\text { SHIVANA } \\
\text { ND }\end{array}$} \\
\hline $\begin{array}{l}\text { R.SRINIVA } \\
\mathrm{S}\end{array}$ & & & & & & & & & & 96 & & & & $\begin{array}{l}10 \\
0\end{array}$ & & & \\
\hline AKINBAMI & & & & & & & & & & $\begin{array}{l}87 . \\
5\end{array}$ & $\begin{array}{l}10 \\
0\end{array}$ & 80 & 80 & & & & \\
\hline $\begin{array}{l}\mathrm{I}=\mathrm{Inf} \\
\mathrm{C}=\mathrm{Cy} \\
\mathrm{B}=\mathrm{B} \\
\mathrm{M}=\mathrm{M}\end{array}$ & $\begin{array}{l}\mathrm{nm} \\
\text { ic } \\
\mathrm{ng} \\
\text { ign }\end{array}$ & ory & & & & ra & $\begin{array}{l}= \\
\mathrm{H}\end{array}$ & $\begin{array}{l}\text { hoic } \\
=\mathrm{I} \\
\text { recl }\end{array}$ & $\begin{array}{l}\text { cho } \\
\text { ic } \\
\text { xed }\end{array}$ & & & & & & & & \\
\hline
\end{tabular}

EVIDENCE LEVEL OF SELECTED ARTICLE TABLE

\begin{tabular}{|l|l|l|c|}
\hline S. No & Author and year & \multicolumn{1}{|c|}{ Article } & Evidence level \\
\hline 1 & R Chandak*, et al 2011 & $\begin{array}{l}\text { An evaluation of efficacy of ultrasonography } \\
\text { in the diagnosis of head and neck swellings }\end{array}$ & 4 \\
\hline 3 & $\begin{array}{l}\text { Shivanand B BAgewadi et al } \\
2010\end{array}$ & $\begin{array}{l}\text { Ultrasonography os swelling in orofacial } \\
\text { region }\end{array}$ & 4 \\
\hline 4 & K Srinivaset . a 20091 & $\begin{array}{l}\text { Ultrasonographic evaluation of inflammatory } \\
\text { swelling of buccal space }\end{array}$ & 4 \\
\hline
\end{tabular}

Summation of Tables

Sensitivity

\begin{tabular}{|l|l|l|l|}
\hline TYPE OF LESION & $\begin{array}{l}\text { R.Chandak } \\
\%\end{array}$ & $\begin{array}{l}\text { K.Srinivas } \\
\%\end{array}$ & B.OAkinbami \\
\hline INFLAMMATORY & 97.1 & 96 & 87.5 \\
\hline CYSTIC & 100 & - & 100 \\
\hline BENIGN & 100 & - & 80 \\
\hline MALIGNANT & 100 & - & 50 \\
\hline
\end{tabular}

Specificity

\begin{tabular}{|l|l|l|l|}
\hline TYPE OF LESION & $\begin{array}{l}\text { R.CHANDAK } \\
\%\end{array}$ & $\begin{array}{l}\text { K.SRNIVAS } \\
\%\end{array}$ & $\begin{array}{l}\text { B.O AKINBAMI } \\
\%\end{array}$ \\
\hline INFLAMMATORY & 100 & 100 & - \\
\hline CYSTIC & 98.3 & - & - \\
\hline BENIGN & 98.3 & - & - \\
\hline MALIGNANT & 98.4 & - & - \\
\hline
\end{tabular}


Ultrasonographic Characterization Of Jaw Swellings- A Systematic Review

POSITIVE PREDICTION

\begin{tabular}{|l|l|l|l|}
\hline TYPE OF LESION & $\begin{array}{l}\text { R.CHANDAK } \\
\%\end{array}$ & $\begin{array}{l}\text { K.SRINIVAS } \\
\%\end{array}$ & $\begin{array}{l}\text { B.O AKINBAMI } \\
\%\end{array}$ \\
\hline INFLAMMATORY & 100 & - & 77.8 \\
\hline CYSTIC & 88.8 & - & 100 \\
\hline BENIGN & 90.2 & - & 80 \\
\hline MALIGNANT & 94.7 & - & 50 \\
\hline
\end{tabular}

NEGATIVE PREDICTION

\begin{tabular}{|l|l|l|l|}
\hline TYPE OF LESION & $\begin{array}{l}\text { R.CHANDAK } \\
\%\end{array}$ & $\begin{array}{l}\text { K.SRINIVAS } \\
\%\end{array}$ & $\begin{array}{l}\text { B.O AKINBAMI } \\
\%\end{array}$ \\
\hline INFLAMMATORY & 97.2 & - & - \\
\hline CYSTIC & 100 & - & - \\
\hline BENIGN & 100 & - & - \\
\hline MALIGNANT & 100 & - & - \\
\hline
\end{tabular}

ACCURACY

\begin{tabular}{|l|l|l|l|}
\hline TYPE OF LESION & $\begin{array}{l}\text { R.CHANDAK } \\
\%\end{array}$ & $\begin{array}{l}\text { K.SRINIVAS } \\
\%\end{array}$ & $\begin{array}{l}\text { B.O AKINBAMI } \\
\%\end{array}$ \\
\hline INFLAMMATORY & 98.5 & - & 70 \\
\hline CYSTIC & 98.5 & - & 100 \\
\hline BENIGN & 98.5 & - & 100 \\
\hline MALIGNANT & 98.5 & - & 100 \\
\hline
\end{tabular}

\section{Inflammatory swellings}

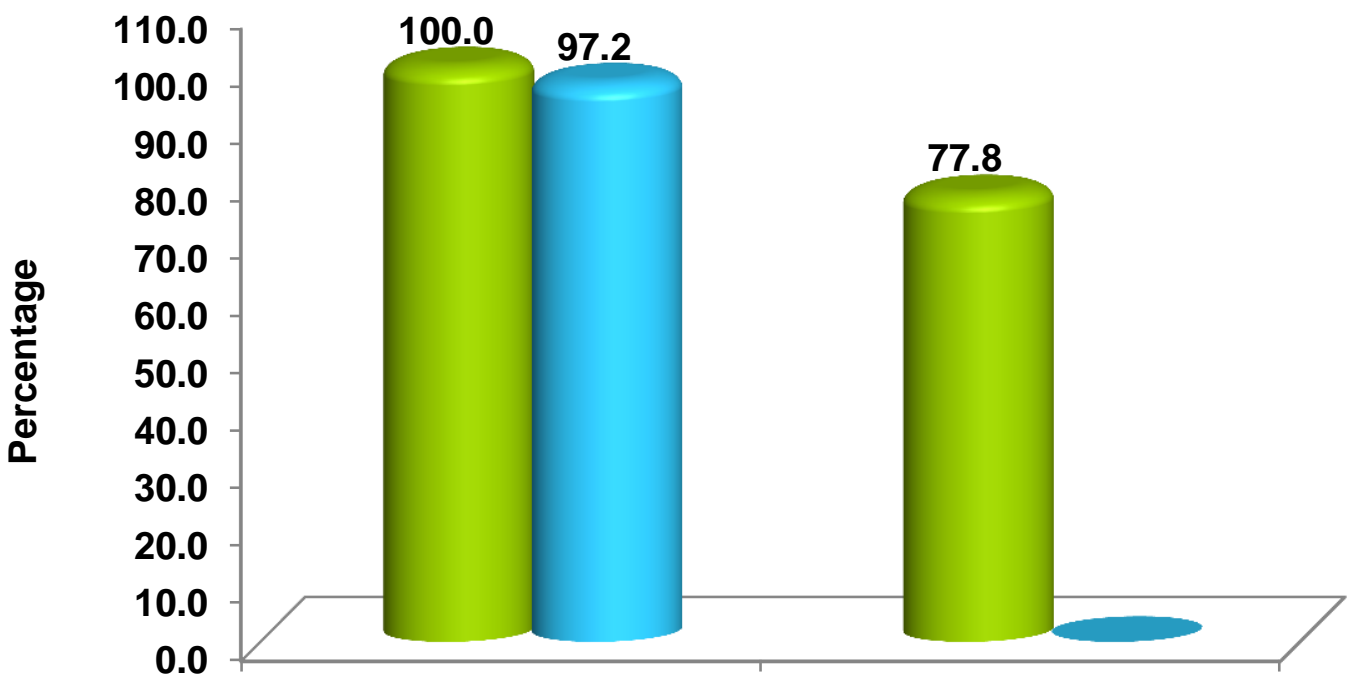

Chandak et, al. 2011

Akinboni et, al. 2006

Akinbami et,al. 2006 Akinbami et,al. 2006 


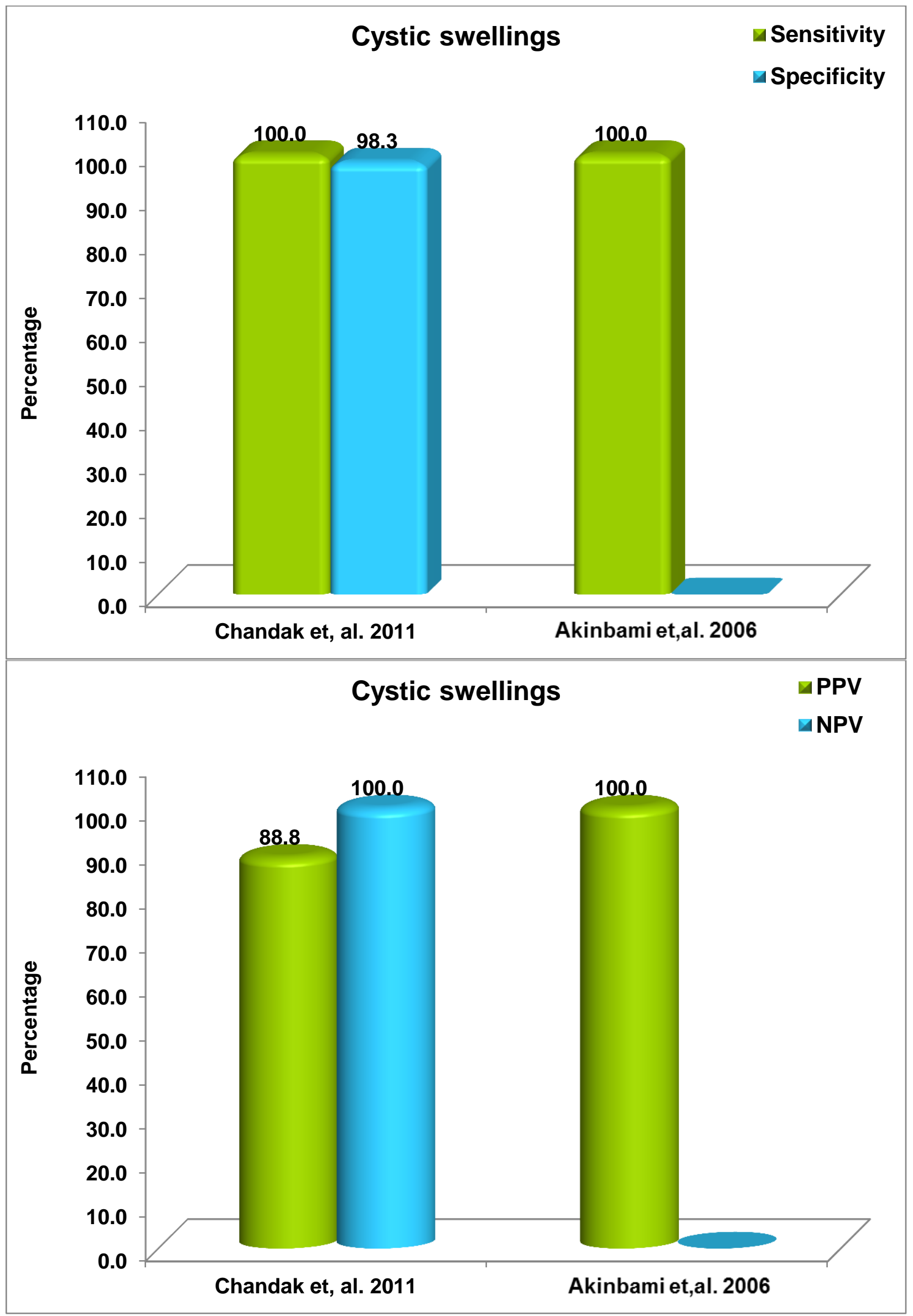




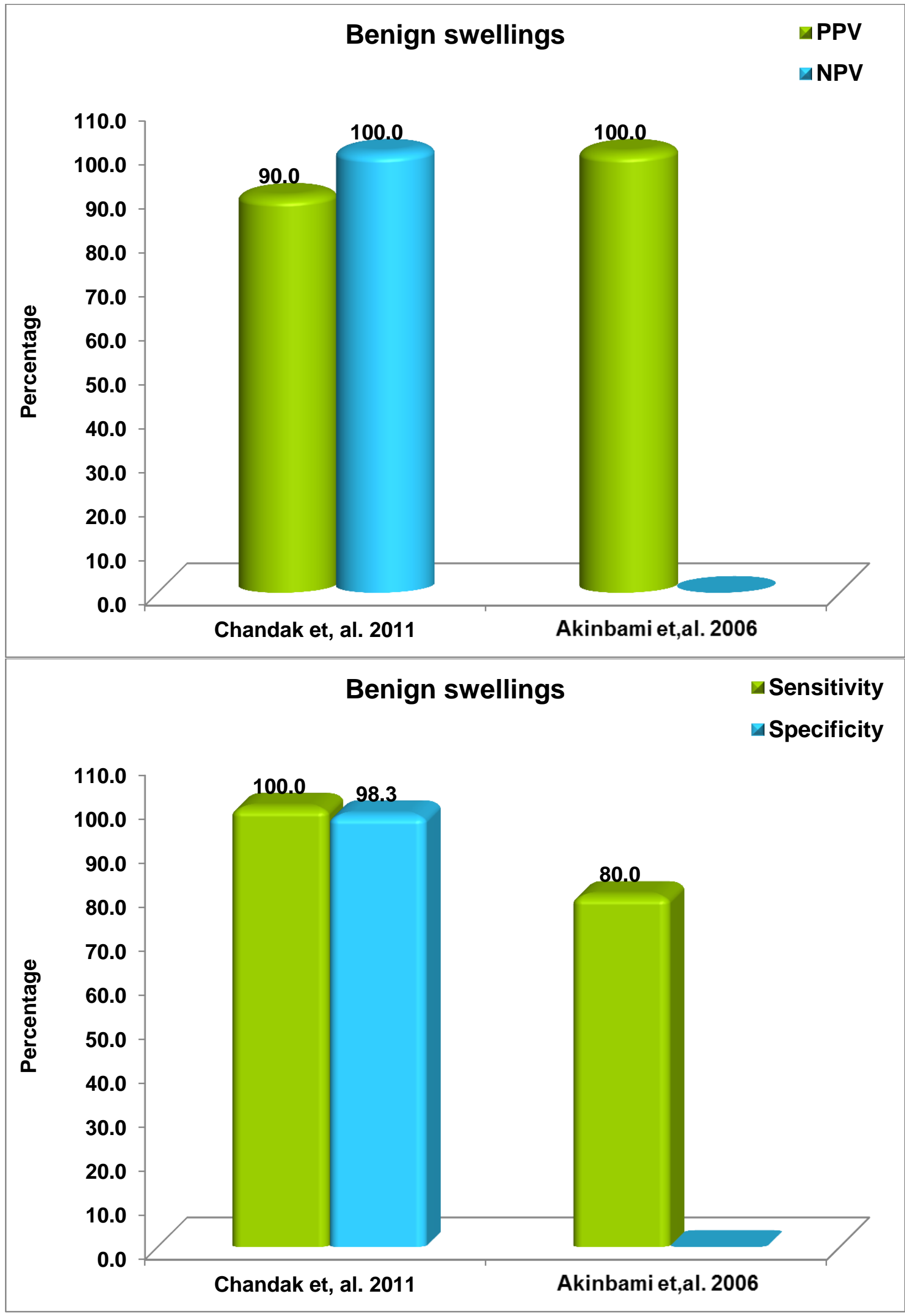




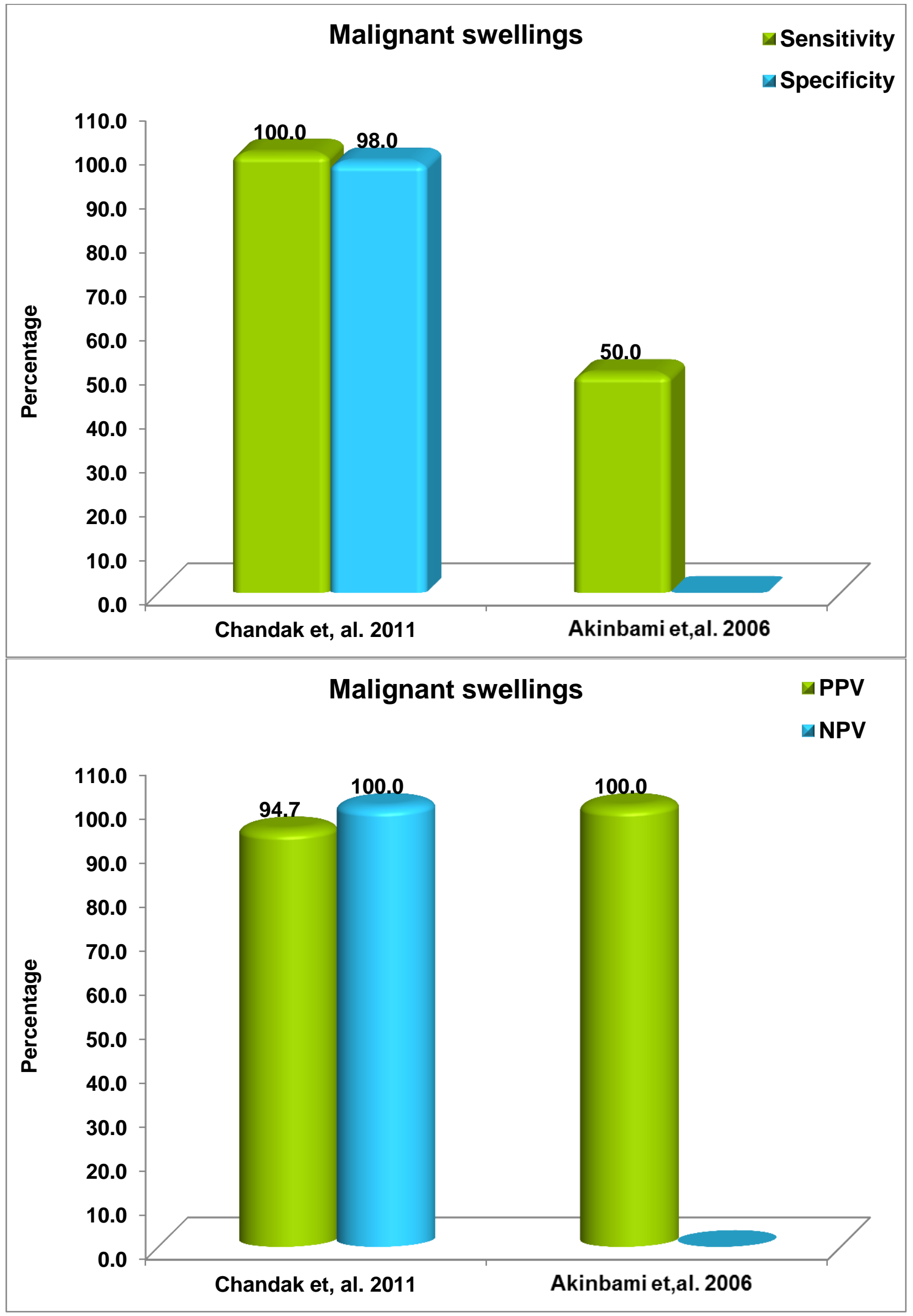


Accurate Reporting and Interpretation of Result

\section{Discussion}

In R.Chandakstudy, most of the inflammatory swellings hadrelatively clear boundaries, irregular shapes, hypoechoicecho intensity and homogeneous ultrasound architectureof lesion.hypoechoic areas and heterogeneous echo texture of thegland as seen in inflammatory swellings in the group of inflammatory swellings,clinical diagnosis had a sensitivity and specificity of $85.7 \%$ whereas sonographic diagnosis had a sensitivityof $97.1 \%$ and specificity of $100 \%$ Cysts on the sonogram appear as anechoic with avery clear boundary and homogeneous echo texture.If the cysts become infected then the content of thelesion can produce some echoes, producing hypoechoicstructures. All cystic lesions showed very clear boundaries, were suggestive of periapical cyst.

In the group of cystic swellings, clinicaldiagnosis had a sensitivity of $75 \%$ and a specificity of $87.1 \%$, whereas sonographic diagnosis had a sensitivityof $100 \%$ and a specificity $98.3 \%$.Clinical diagnosis of benign neoplasmshad a sensitivity of $77.7 \%$ and specificity of $86.8 \%$, whereas sonographic diagnosis had a sensitivity of $100 \%$ and a specificity $98.3 \%$, and the accuracy of thetest was $98.5 \%$ Ultrasound can predict malignancy in $89 \%$ of cases butvarious forms of malignancy cannot be differentiated. Onultrasounds of lower grade tumours, smaller lesions mayappear as well defined and similar to a benign tumour.malignantneoplasms, clinical diagnosis had a sensitivity of $94.4 \%$ and specificity of $82.6 \%$, whereas sonographic diagnosishad a sensitivity of $100.0 \%$ and specificity of $98.0 \%$.

InShivanand B. Bagawadiet al ultrasonography diagnosis inflammatory swellings was anechoic /hypoechoic pattern with clinical diagnosis $100 \%$ and ultrasonographic diagnosis of $100 \%$,cystic swellings was anechoic pattern with clinical diagnosis of $96.6 \%$ and USG of $100 \%$, benign swellings shows hypoechoic pattern with clinical diagnosis of $100 \%$ and USG of $100 \%$,malignant swellings was hypo/hyperechoic pattern with clinical diagnosis of $100 \%$ and USG of $100 \%$.

According to R.Srinivaset al inflammatory swellings of buccal space in USG shows hypoechoic in $54.2 \%$ and anechoic in $45.8 \%$,Clinical diagnosis was $92 \%$ and USG was $96 \%$ and sensitivity of clinical criteria over ultrasonographic diagnosis was $96 \%$ with a specificity of $100 \%$.

In B.O Akinbamiet al inflammatory swellings shows accuracy $70 \%$,sensitivity $87.5 \%$,specificity $0.0 \%$ positive prediction was $77.8 \%$ and negative prediction was $0.0 \%$.

In cystic swellings the accuracy is $100 \%$,sensitivity $100 \%$,specificity $0.0 \%$,positive prediction $100 \%$, negative prediction $0.0 \%$

In benign swellings accuracy is $80 \%$,sensitivity $80 \%$,specificity $0.0 \%$ positive prediction $100 \%$ and negative prediction is $0.0 \%$

In malignant swellings the accuracy is $50 \%$,sensitivity $50 \%$,specificity $0.0 \%$ positive prediction $100 \%$ and negative prediction was $0.0 \%$.

Quality assurance TABLE STARD Statement

Standards for the Reporting of Diagnostic accuracy studies

\begin{tabular}{|l|l|l|l|l|}
\hline Check List & R.Chandak & Shivanand & K.Srinivas & BO Akinmami \\
\hline Inclusion, exclusion criteria & Mentioned & Mentioned & Mentioned & Mentioned \\
\hline $\begin{array}{l}\text { Compared with gold } \\
\text { standard }\end{array}$ & No & Yes & No & Yes \\
\hline $\begin{array}{l}\text { Described about data } \\
\text { collection }\end{array}$ & No & No & No & No \\
\hline $\begin{array}{l}\text { Described reference } \\
\text { standard and rationale }\end{array}$ & No & Yes & No & Yes \\
\hline Units rationale & Mentioned & Mentioned & Mentioned & Mentioned \\
\hline $\begin{array}{l}\text { Training and expert of } \\
\text { person }\end{array}$ & Mentioned & not mentioned & Not mentioned & Mentioned \\
\hline Blinding & Mentioned & No & No & No \\
\hline Satistical methods CI\% & No & No & No & No \\
\hline $\begin{array}{l}\text { Results -Test } \\
\text { Reproducibility }\end{array}$ & Mentioned & Not mentioned & Not mentioned & Not mentioned \\
\hline $\begin{array}{l}\text { Flow chart for clinical } \\
\text { criteria }\end{array}$ & No & No & No & No \\
\hline
\end{tabular}


Ultrasonographic Characterization Of Jaw Swellings- A Systematic Review

\begin{tabular}{|l|l|l|l|l|}
\hline $\begin{array}{l}\text { Treatment done in time and } \\
\text { interval of and reference std }\end{array}$ & No & No & No & No \\
\hline Participants target condition & Mentioned & Mentioned & Mentioned & Mentioned \\
\hline Tabulation of results & Mentioned & Mentioned & Mentioned & Mentioned \\
\hline Outlier data & Not mentioned & Not mentioned & Not mentioned & Not mentioned \\
\hline $\begin{array}{l}\text { Clinical applicability of } \\
\text { study }\end{array}$ & Mentioned & Mentioned & Mentioned & Mentioned \\
\hline
\end{tabular}

\section{Conclusion}

Ultrasonography can be used as a diagnostic aid in jaw swellings.But Quality studies which assesing the diagnostic accuracy, sensitivity and specificity are less.Among three studies, all studies gives high sensitivity, specificity for inflammatory and cystic swellings. Whereas sensitivity and specificity in assesing for benign and malignant lesions, the studies shows highly variable results.To conclude, quality studies are needed to establish whether the ultrasonography diagnosis is accurate in all jaw swellings.

\section{INCLUDED STUDIES}

\section{References}

[1]. R Chandak, SDegwekar, RR Bhowte, M Motwani, P Banode, M Chandak and S Rawlani- Department of Oral medicine and Radiology An evaluation of efficacy of ultrasonography in the diagnosis of head and neck swellings- journal of Dentomaxillofacial Radiology (2011)40, 213-221(2011 The British Institute of Radiology)

[2]. Shivanand B Bagewadi, Mahima VG, KarthikeyaPatil. Ultrasonography of Swellings in Orofacial Region. JIAOMR 2010;22(1):1826.

[3]. K.Srinivas, KN Sumanth, ss Chopra- Department of oral medicine and Radiology,Oxford Dental college and hospital, Bangalore, ultrasonographic evaluation of inflammatory swellings of buccal space-Indian journal of dental research,20(4), 2009

[4]. B.O Akinbami,* V.I Ugboko F.J. Owotade, A.E. Obiechina, V.O. Adetiloye and O.Ayoola-Department of oral and maxillofacial surgery and Radiology Applications of ultrasonography in the diagnosis of soft tissue swellings of the cervicofacial region-journal of WAJM vol, 25 No 2,2006. 